\title{
COMMENT
}

\section{Designing biofunctional immunotherapies}

\author{
Elyse A. Watkins and Jeffrey A. Hubbell*
}

Intelligent design of materials for biomedical applications involves the development of technologies that are informed by an understanding of biological systems. Immunoengineers have been making essential inroads in developing therapeutics endowed with designed biofunctionality, particularly in vaccinology, cancer immunotherapy and immune tolerance.

\section{Vaccines}

The first modern notion of vaccines arose in the late 18 th century for the treatment of smallpox. Since then, over 80 vaccines have been approved for use in the United States. Many of these vaccines are live attenuated vaccines - they include the organism against which an immune response should be targeted, grown in suboptimal conditions or mutated to not cause disease. Although highly effective, live attenuated vaccines are complex, require a long time for development, are difficult to develop for rapidly mutating and chronic viruses and may have difficulty in maintaining stability.

Vaccine development has been shifting towards designing vaccines with materials and molecules that synthetically recapitulate the immunogenic properties of live attenuated vaccines, such as the generation of broadly neutralizing antibodies and robust memory responses, while avoiding their drawbacks. These vaccines contain components of the pathogen and adjuvants to activate an immune response. For the design of biofunctional vaccines, their spatial and temporal bioavailability, the targeted antigen presenting cells (APCs) and the adjuvants used to maximally stimulate APCs while avoiding toxicity must be considered.

The route of administration and drainage to lymph nodes are important aspects for the bioavailability of vaccines. For example, most vaccines are administered intramuscularly, subcutaneously or intradermally, whereas many pathogens infect at mucosal sites, which exhibit a distinct immune response that is different from that in the muscle or skin. Intranasal administration is particularly effective in eliciting an immunoglobulin A (IgA) antibody response important for pathogen clearance at mucosal sites; however, materials are required that enable agent escape from mucus, resistance to proteases and penetration through epithelial barriers to allow widespread clinical translation of mucosal vaccines.

There are multiple routes for a vaccine to enter the lymph node. APCs around the site of injection can acquire the vaccine and actively travel to the lymph node to prime $\mathrm{T}$ cells. Alternatively, vaccines can passively enter the lymphatics through fluid drainage. For example, optimal passive lymph node targeting can be achieved using sub-50 nm poly(ethylene glycol)-stabilized poly(propylene sulfide) nanoparticles, which are delivered to the lymphatics and retained by APCs in the lymph nodes ${ }^{1}$.

Viruses and live attenuated vaccines persist for an extended period of time in the host, which elicits a robust immune response. Therefore, another important consideration to ensure bioavailability of designed vaccines is the duration of antigen persistence. Current vaccines are primarily bolus injections and thus may be cleared before mounting a sufficient immune response. Multiple injections of vaccines or osmotic pumps can be used to extend antigen exposure and thus substantially increase antibody responses compared with bolus injection alone ${ }^{2}$. Similarly, a material allowing slow release of antigen, or smart dosing strategies, could lead to more robust antibody responses.

Targeting specific subsets of cells, in particular dendritic cells and B cells, while avoiding over-activation and toxicity is an important consideration in vaccine design. Our lab developed a vaccine that contains a mannose polymer conjugated to a Toll-like receptor 7 (TLR7) agonist and the antigen of interest. The mannose polymer is rapidly endocytosed through recognition by lectins on the surface of cross-presenting and migratory dendritic cells, which subsequently activate cytotoxic and helper $\mathrm{T}$ cells, avoiding systemic over-activation compared with unconjugated adjuvant ${ }^{3}$. Targeting B cells is important to induce robust responses, and it is widely accepted that vaccines with high multivalency, such as virus-like particles and nanoparticles with high antigen density on the surface, can directly act on B cell receptors to promote humoral responses.

Adjuvants are essential components of vaccines, yet most vaccines that are clinically approved in the United States contain adjuvants that were developed with limited mechanistic insight. In the past 10 years, progress towards adjuvant biofunctionality has been made in the clinical approval of monophosphoryl lipid A, a TLR4 agonist, and CpG, a TLR9 agonist, which both bind to 
innate immune receptors and trigger downstream activation in APCs. Although this is an important advance, it is only scratching the surface. New adjuvants should probe a variety of pattern recognition receptors and pathways that may be triggered to activate APCs, for example, C-type lectins, nucleotide-binding oligomerization domain (NOD)-like receptors, retinoic acidinducible gene-I-like (RIG)-I, the inflammasome and the stimulator of interferon genes (STING) pathway.

Many vaccines currently in development are limited in their ability to create durable memory responses, broadly neutralizing antibodies and cytotoxic $\mathrm{T}$ cell responses. Intelligent design of vaccines will learn from biology to develop biofunctional materials.

\section{Cancer immunotherapy}

Cancer immunotherapy faces similar requirements and challenges as vaccinology: the need to mount robust and durable immunity. However, tumours pose additional hurdles owing to their immunosuppressive microenvironment, which suppresses T cell activity, is constantly changing to avoid immune recognition and contains mainly 'self'-antigens to which the immune system is robustly tolerized. Furthermore, tumour cells are heterogeneous not only among patients but also within a tumour, making it difficult to develop a therapy that targets a single antigen or inflammatory pathway. Cancer immunotherapies should aim at reversing the suppressed phenotype of tumour-specific T cells to activate them, and avoid toxicity associated with systemic over-activation.

Early efforts to promote immune-mediated killing of tumour cells sought to reverse the suppressed phenotype of tumour-specific T cells by globally activating $\mathrm{T}$ cells. The discovery of immune checkpoints and the development of corresponding inhibitors were a breakthrough in immunotherapy owing to their ability to reverse the suppressed phenotype of T cells and thus destroy tumours in some patients, particularly in patients with inflamed tumours. Other strategies, such as cytokine therapy, also aim at broadly activating APCs and T cells, but they often lead to off-target liver toxicity and even autoimmunity.

To achieve tumour-specific activation, therapies can be directly delivered to the tumour microenvironment to activate local T cells. Alternatively, predefined tumour antigens can be systemically administered together with adjuvants. By conjugating the checkpoint inhibitors anti-cytotoxic T lymphocyte antigen 4 (CTLA4) and anti-programmed cell death 1 ligand 1 (PD-L1) to peptides with a high affinity for the extracellular matrix surrounding a tumour, our lab demonstrated that inhibitors remain in the tumour microenvironment, activate local $\mathrm{T}$ cells, reduce the tumour burden and minimize off-target effects ${ }^{4}$. An immune-activating microenvironment can also be created by activating the intracellular STING pathway, which senses nucleic acid and protects against viral infection and cancer. For example, intratumourally delivered polymersomes can escape the endosomal compartment to release a cyclic dinucleotide STING agonist in the cytoplasm of APCs and induce the expression of type I interferons ${ }^{5}$. The interferons then activate cytotoxic T cells, leading to a nearly complete regression of aggressive tumours in mice.

Numerous strategies also exist to systemically deliver tumour-specific cancer vaccines; for example, exploiting the natural property of serum albumin accumulation in the lymph node by using molecules that contain a lipid tail that binds to endogenous albumin upon injection in vivo, and a tumour antigen or the adjuvant $\mathrm{CpG}$. Following administration, the molecules accumulate in the lymph nodes and are phagocytosed by APCs, leading to the activation of tumour antigen-specific T cells and tumour regression. Alternatively, the physical properties of liposomes can be modulated to selectively target dendritic cells in the spleen and lymph nodes after intravenous delivery ${ }^{6}$. The liposomes can be used to deliver tumour antigen-encoded RNA, which serves as a TLR7 agonist, triggering dendritic cell activation, expression of antigen, robust activation of $\mathrm{T}$ cells and complete regression of advanced tumours.

\section{Tolerogenic vaccination}

Eliciting anti-pathogen and anticancer immunity requires robust immune activation. By contrast, autoimmune disease, organ transplantation, allergy and antidrug immunity require immune suppression. Treatments for these conditions include broadly immunosuppressive agents such as steroids, blocking inflammatory cytokines and lymphocyte depletion. These treatments require a lifetime of immunosuppression and predisposition of patients to opportunistic infections and other comorbidities. Therefore, there is an unmet need for antigen-specific tolerance, or tolerogenic vaccines, and immunologists and immunoengineers have been working on strategies to achieve this goal over the past decade.

First generation antigen-specific immunosuppressive approaches relied on the systemic administration of antigenic peptides that are taken up by APCs, which, in the absence of inflammatory signals, leads to $\mathrm{T}$ cell deletion and tolerance. This strategy continues to be a potential therapy in the clinic; however, cases of anaphylactic reactions in patients with ongoing immune responses and the need to know a predefined autoimmune peptide have limited their use. Pioneers in the field of antigenspecific tolerance conjugated autoantigens to fixed apoptotic cells, and found that APCs take up the autoantigens and process them in the same way as they process apoptotic debris - in a tolerogenic manner ${ }^{7}$. To minimize the use of labour-intensive and cost-intensive cell therapy, materials and molecules have been developed that can accomplish the same goal of targeting the apoptotic pathway. Our lab reasoned that by intravenous injection of molecules that bind to red blood cells, which turn over at a rate of $1 \%$ per day in humans, we could target a large amount of apoptotic cells. Indeed, targeting antigen to red blood cells leads to $\mathrm{T}$ cell deletion and exhaustion and prevents diabetes in a mouse model ${ }^{8}$. Alternatively, APCs can be directly targeted to achieve T cell tolerance by modulating the size, charge and targeting motifs of molecules carrying an autoimmune antigen payload.

In these models of prophylactic tolerance, there is no ongoing immune response. However, during an ongoing 
immune response, such as in autoimmune disease or during the development of antidrug antibodies, activated B cells, memory B cells and plasma cells are at play. Thus, protein drug treatments are likely to be opsonized by antibodies and rendered ineffective. Moreover, after a $B$ cell response has been initiated, $T$ cell tolerance may be insufficient to control the ongoing $B$ cell response. Therefore, understanding B cell biology is key to finding a strategy for antigen-specific B cell deletion. For example, it is known that co-ligation of CD22, which is a sialic acid binding protein on $\mathrm{B}$ cells, and the $\mathrm{B}$ cell receptor triggers B cell apoptosis. Thus, liposomes coated with a specific antigen and an anti-CD22 antibody can elicit B cell deletion?.

Although there has been enormous progress in the field in the past decade, major challenges remain to achieve antigen-specific tolerance. First, many preclinical studies have shown that tolerance is achieved in a prophylactic setting; however, in most circumstances immunity pre-exists, and memory and activated lymphocytes as well as antibodies in the circulation constitute considerable challenges to overcome. Second, the diversity of human leukocyte antigens (HLA) and of the peptides presented to T cells makes it difficult to design treatments that prevent transplant rejection in HLA-mismatched patients. Finally, a fundamental understanding of the development of regulatory cells, in particular of regulatory $\mathrm{T}$ cells, will be needed to design therapies that maximize regulatory $\mathrm{T}$ cell development and retain their phenotype to provide lasting dominant tolerance that can suppress reactions to the multiple antigens involved in autoimmune diseases, some of which are known and others of which are not. Immunoengineers will have to closely collaborate with immunologists to overcome these challenges.

1. Reddy, S. T. et al. In vivo targeting of dendritic cells in lymph nodes with poly(propylene sulfide) nanoparticles. J. Control. Release 112 , 26-34 (2006).

2. Tam, H. H. et al. Sustained antigen availability during germinal center initiation enhances antibody responses to vaccination. Proc. Natl Acad. Sci. USA 113, E6639-E6648 (2016).

3. Wilson, D. S. et al. Antigens reversibly conjugated to a polymeric glyco-adjuvant induce protective humoral and cellular immunity. Nat. Mater. 18, 175-185 (2019).

4. Ishihara, J. et al. Matrix-binding checkpoint immunotherapies enhance antitumor efficacy and reduce adverse events. Sci. Trans/ Med. 9, eaan0401 (2017).

5. Shae, D. et al. Endosomolytic polymersomes increase the activity of cyclic dinucleotide STING agonists to enhance cancer immunotherapy. Nat. Nanotechnol. https://doi.org/10.1038/ s41565-018-0342-5 (2019).

6. Kranz, L. M. et al. Systemic RNA delivery to dendritic cells exploits antiviral defence for cancer immunotherapy. Nature 534, 396-401 (2016).

7. Luo, X. et al. ECDI-fixed allogeneic splenocytes induce donorspecific tolerance for long-term survival of islet transplants via two distinct mechanisms. Proc. Natl Acad. Sci. USA 105 , 14527-14532 (2008).

8. Kontos, S. et al. Engineering antigens for in situ erythrocyte binding induces T cell deletion. Proc. Natl Acad. Sci. USA 110, E60-E68 (2013).

9. Pang, L. et al. Encapsulating an immunosuppressant enhances tolerance induction by siglec-engaging tolerogenic liposomes. Chembiochem 18, 1226-1233 (2017).

\section{Acknowledgements}

J.A.H. receives research funding from the Juvenile Diabetes Research Foundation (JDRF) on tolerogenic vaccines.

\section{Competing interests}

J.A.H. founded, owns equity in and receives research funding from Anokion, Inc. and Kanyos Bio, Inc., which are developing tolerogenic vaccines based on patents filed by his university, upon which he and E.A.W. are co-inventors. J.A.H. founded and holds equity in Arrow Immune, Inc., which is developing immuno-oncology drugs based on patents filed by his university, upon which he is co-inventor. J.A.H. is also co-inventor on university patents on nanomaterial vaccines. 